DOI: $12737 / 25212$

УДК 631.0.33: 634.958

\author{
СОВЕРШЕНСТВОВАНИЕ ТЕХНОЛОГИЧЕСКОГО ОБОРУДОВАНИЯ МАШИНЫ ДЛЯ ОБРЕЗКИ \\ КРОН ДЕРЕВЬЕВ В ПРИДОРОЖНЫХ ЛЕСНЫХ ПОЛОСАХ \\ кандидат технических наук В. П. Попиков ${ }^{1}$ \\ кандидат технических наук, доцент Л. Д. Бухтояров ${ }^{1}$ \\ 1 - ФГБОУ ВО «Воронежский государственный лесотехнический университет имени Г.Ф. Морозова», \\ г. Воронеж, Российская Федерация
}

При рубках ухода при обрезке ветвей деревьев в придорожных и полезащитных лесных полосах широко применяются обрезчики контурные предназначенные для сплошной горизонтальной, вертикальной и наклонной обрезки крон. Эти устройства являются навесными или съемными и агрегатируются с широко распространенными в лесном хозяйстве колесными тракторами тяговых классов 0,6-1,4 кН. Совершенствование конструкций таких устройств идет по следующим основным направлениям: срезающим устройствам и гидроприводу. В предлагаемом рабочем органе машины для обрезки крон деревьев, содержащем базовую машину, гидроманипулятор на рукояти которого установлен

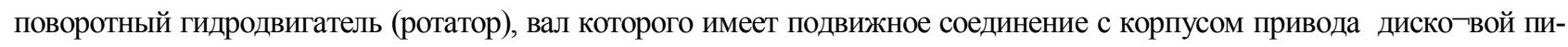
лы, имеющей одностороннюю заточку в сторону отделяемой части ветви, V-образный упор выполнен в виде гидроцилиндра одностороннего действия с подпружиненным штоком, поршневая полость которого подсоединена последовательно к линии слива гидромотора, в которую установлен регулируемый дроссель для создания давления рабочей жидкости (подпора). При таком выполнении устройства при подводе дисковой пилы к срезаемой ветви V- образный упор с подпружиненным штоком выдвигается до упора в срезаемую ветвь и исключает зажим дисковой пилы в пропиле, что позволит повысить надежность и производительность. В статье была разработана математическая модель устройства для обрезки крон деревьев с гидроприводом дисковой пилы на основе общей методики моделирования плоских механизмов. Были составлены дифференциальные уравнения процесса резания. Разработанная модель предлагаемой конструкции устройства для обрезки крон деревьев позволяет изучить влияние геометрических и механических параметров ветвей деревьев, технологических параметров процесса резания на энергозатраты и качество среза, с учетом конструктивных параметров. Модель позволяет также оценить работоспособность и изучить эффективность применения исследуемого устройства в различных условиях эксплуатации.

Ключевые слова: рабочие процессы, обрезка, трактор, ветви деревьев, гидропривод, манипулятор, дисковая пила.

\title{
IMPROVEMENT OF TECHNOLOGICAL EQUIPMENT OF THE MACHINE FOR TRIMMING TREE CROWNS IN ROADSIDE FOREST BELTS
}

$\mathrm{PhD}$ in Engineering V. P. Popikov ${ }^{1}$

$\mathrm{PhD}$ in Engineering, Associate Professor L. D. Bukhtoyarov ${ }^{1}$

1 - Federal State Budget Education Institution of Higher Education «Voronezh State University of Forestry and Technologies named after G.F. Morozov», Voronezh, Russian Federation

\begin{abstract}
When cleaning cutting, pruning of branches of roadside trees and shelter belt contour cutters are widely used, cutters is designed for total horizontal, vertical and oblique trimming crowns. These devices are hinged or removable ones and aggregated with wheeled tractors of traction class 0.6 to $1.4 \mathrm{kN}$, widespread in forestry. Improving the design of such devices is made in the following areas: cutting devices and hydraulic drives. In the proposed working body of the machine for cutting tree crowns containing base machine, crane on the handle of which a rotary hydraulic motor (rotator)is mounted, the shaft of which has movable connection with the housing of the circular saw with one-sided sharpening in the direction of the detachable part of the branch, V-shaped emphasis in the form of unilateral action hydraulic cylinder with spring-loaded rod, piston cavity which is connected in
\end{abstract}


series with the drain lines of the hydraulic motor which is mounted an adjustable throttle to create pressure of the working fluid (support). In this implementation of the device when circular goes deep into the branch, which is cut, the $\mathrm{V}$ - shaped support with spring-loaded rod moves all the way in the branch and eliminates the clamping of the saw blade in the cut, which will improve reliability and performance. The article has developed a mathematical model of device for pruning tree crowns by circular saw with hydraulic drive on the basis of common methodology for the simulation of planar mechanisms. Differential equations of the cutting process were composed. The model of proposed design of the device for cutting tree crowns allows to study the influence of geometrical and mechanical parameters of the branches of the trees, technological parameters of cutting process on energy consumption and quality of the cut, taking into account design parameters. The model allows also to assess the performance and to examine the effectiveness of the device in different operating conditions.

Keywords: workflows, cutting, tractor, tree branches, hydraulic drive, crane, circular saw.

Одним из важнейших лесоводственных мероприятий в процессе выращивания и эксплуатации придорожных насаждений являются рубки ухода. В различные периоды жизни они включают: осветление и прочистку с вырубкой отдельных экземпляров деревьев, обрезку боковых ветвей и сухих вершин, обрезку кустарников и т.д. $[1,2]$. Известен обрезчик контурный механизированный ОКМ-4,5 предназначенный для сплошной горизонтальной, вертикальной и наклонной обрезки крон плодовых деревьев. Он может быть использован для контурной обрезки декоративных насаждений и полезащитных и придорожных лесополос, агрегатируется с трактором Т-74, оборудованным ходоуменьшителем.

Обрезчик МКО-3 (рис. 1) состоит из системы навески, опорной и подъемной рам, дискового режущего аппарата, защитного ограждения кабины трактора и гидросистемы.

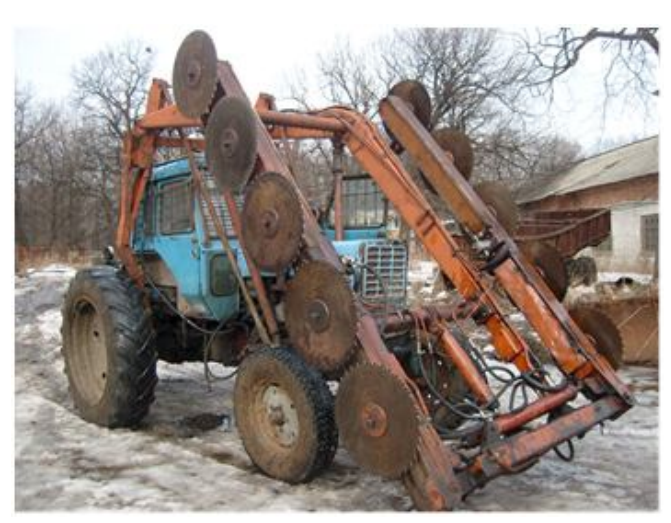

Рис. 1. Обрезчик контурный механизированный МКО-3

Дисковый режущий аппарат имеет сварную раму, внутри которой размещены валы привода пил, клиноремённая передача и устройство для ее регули- ровки. В центральной части рамы установлен гидродвигатель МНШ-46У привода пил.

Во ВНИИАЛМИ для срезания сухих вершин деревьев в лесных полосах разработаны несколько срезающих механизмов с дисковыми пилами, смонтированных на стреле экскаватора ЭО-2621 вместо ковша. Существующие конструкции машин для обрезки ветвей и вершин деревьев обеспечивают высоту не более $4,5 \mathrm{~m}$, в то время как на лесосеменных плантациях и участках требуемая высота обрезки 7-8 м, кроме того они не исключают зажима дисковой пилы в пропиле $[3,4,5]$.

Предлагаемое нами изобретение решает задачу повышения эффективности подрезки крон деревьев и позволяет достигнугь технического результата - при подводе дисковой пилы к срезаемой ветви $\mathrm{V}$ - образный упор с подпружиненным штоком выдвигается до упора в срезаемую ветвь и исключает зажим дисковой пилы в пропиле [7].

Для этого в рабочем органе машины для обрезки крон деревьев, содержащем базовую машину, гидроманипулятор на рукояти которого установлен поворотный гидродвигатель (ротатор), вал которого имеет подвижное соединение с корпусом привода дисковой пилы, имеющей одностороннюю заточку в сторону отделяемой части ветви, V-образный упор выполнен в виде гидроцилиндра одностороннего действия с подпружиненным штоком, поршневая полость которого подсоединена последовательно к линии слива гидромотора, в которую установлен регулируемый дроссель для создания давления рабочей жидкости (подпора), необходимого для достижения требуемого усилия на штоке гидроцилиндра для устранения зажима дисковой пилы в пропиле.

На рис. 2а схематически изображен рабочий орган машины для обрезки крон деревьев, вид сбоку. На 


\section{Технологии. Машины и оборудование}

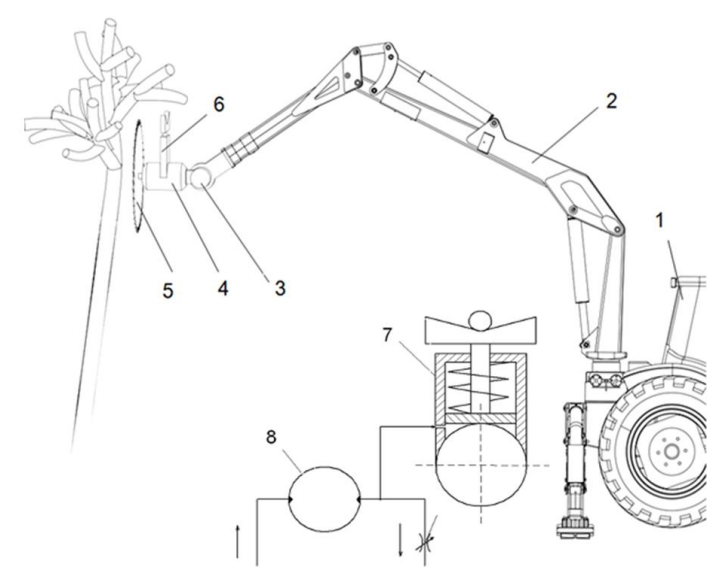

Рис. 2. Обрезчик контурный

$a$ - схема рабочего органа, вид сбоку; 6 - V-

образный упор с подпружиненным штоком

рис. 26 изображен V-образный упор с подпружиненным штоком.

Рабочий орган машины содержит базовую машину 1 , гидроманипулятор 2 , на рукояти которого установлен поворотный гидродвигатель 3 , вал которого соединен с корпусом привода в котором установлен гидромотор 4 , дисковая пила 5 , на корпусе привода установлен V-образный упор 6, жестко соединен с подпружиненным штоком гидроцилиндра 7, поршневая полость которого подсоединена к линии слива гидромотора, в которую установлен регулируемый дроссель 8 для создания подпора рабочей жидкости.

Рабочий орган машины для обрезки крон деревьев работает следующим образом. Базовая машина 1 занимает позицию перед деревом, и оператор при помощи гидроманипулятора 2 поднимает и наводит рабочий орган на ветвь. Включением привода 4 обеспечивается вращение дисковой пилы 5 , затем поочередным манипулированием гидроцилиндрами гидроманипулятора происходит подрезка кроны дерева при неизменном положении базовой машины.

В процессе обрезки крупных боковых ветвей и вершин деревьев давление рабочей жидкости в сливной магистрали 9 достигает определенной величины закрытием регулируемого дросселя 8 для создания подпора. V-образный упор 6 с подпружиненным штоком упирается в отделяемую часть ветви, исключая зажим дисковой пилы в пропиле.

При ежегодной подрезке однолетних побегов используется эта же пила, которая устанавливается на вал гидромотора другой стороной. При обрезке однолетних побегов зуб пилы их как бы захватывает и не дает отклоняться в сторону. В этом случае регулируемый дроссель 8 открыт полностью, не создает подпора рабочей жидкости и подпружиненный выдвигается. Далее рабочий орган переводится в транспортное положение, и базовая машина перемещается на новую позицию для обрезки кроны другого дерева.

Нами проведено обоснование конструктивнотехнологической схемы машины для обрезки крон деревьев и разработка математических моделей рабочих процессов машины. Составлены и проанализированы дифференциальные уравнения, описывающие расход рабочей жидкости гидропривода и рабочий процесс резания дисковой пилой при повороте маятникового рычага поворотным гидродвигателем (ротатором) [6].

$$
\left\{\begin{array}{l}
\frac{\mathrm{dp}}{\mathrm{dt}}=\frac{1}{\mathrm{~K}_{\mathrm{p}}}\left(\mathrm{q}_{\mathrm{n}} \cdot \omega_{\mathrm{n}}-\mathrm{q}_{\mathrm{M}} \cdot \omega-\mathrm{a}_{\mathrm{y}} \cdot \mathrm{p}-\mathrm{q}_{\mathrm{r}} \cdot \varphi_{\mathrm{n}}\right) ; \\
\frac{\mathrm{d} \omega}{\mathrm{dt}}=\frac{1}{\mathrm{~J}_{\text {пр }}}\left\{\frac{\eta_{\mathrm{n}} \mathrm{q}_{\mathrm{m}} \mathrm{p}}{2 \pi \eta_{0}}-\mathrm{k}_{\mathrm{M}} \frac{\mathrm{dN}}{\mathrm{dt}}\left[\mathrm{k}_{\mathrm{n}} \cdot \mathrm{h}_{\text {пог }}+\left(\frac{\mu}{\operatorname{tg} \delta}+1\right) \mathrm{c}_{\text {рез }} \cdot \mathrm{h}_{\text {по }}^{2}\right] \cdot \mathrm{R}_{\mathrm{p}} \cdot \operatorname{sign}(\omega)-\mathrm{k}_{\omega} \omega\right\},
\end{array}\right.
$$

где $\mathrm{p}$ - давление рабочей жидкости в гидросистеме, Па; $\mathrm{t}$ - время, с; Кр - коэффициент податливости упругих элементов гидропривода, м5/H; qн - рабочий объём насоса, м3/об; qм - рабочий объём гидромотора, м3/об; $\omega н$ - угловая скорость вращения насоса, с-1; $\omega$ - угловая скорость вращения вала гидромотора, с-1; ау - коэффициент утечек, м5/(H·c); Јпр- приведенный момент инерции вращающихся масс к валу гидромотора, включающий момент инерции роторной группы гидромотора и момент инерции рабочего органа, кг·м2; ฤ0 - объёмный КПД гидромотора; qг - рабочий объём ротатора, м3/об; фп - угол поворота маятника, рад.; $\mathrm{h}_{\text {под }}=0,12 \cdot \frac{\mathrm{v}_{\text {под }}}{2 \pi \omega}-$ подача на режущий зуб, м; kп - удельное сопротивление перерезанию, H/м2; $\mu$ - коэффициент трения древесины о зуб; $\delta$ - угол резания передней режущей кромки, рад.; срез - коэффициент пропорциональности, постоянный для данной обрабатываемой древесины; Rp - радиус резания, м; k сопротивления резанию. 
Система дифференциальных уравнений (1) решена методом численного интегрирования - модифицированным методом Эйлера-Коши. Для решения системы дифференциальных уравнений, положенной в основу модели и для проведения различных компьютерных экспериментов с моделью составлена компьютерная программа на языке ObjectPascal в интегрированной среде программирования BorlandDelphi 7.0. На экран выводятся графики угловой скорости и давления на гидромоторе пилы (рис. 3).
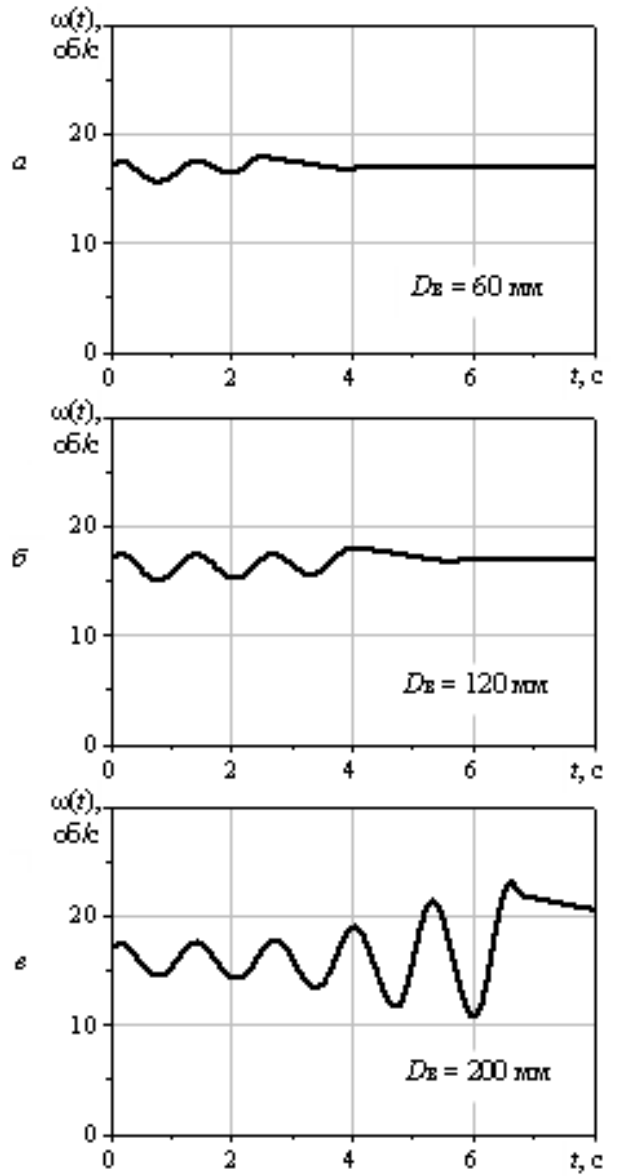

Рис. 3. Временные зависимости угловой скорости диска $\omega$ при различных диаметрах ветви

Для оценки влияния диаметра ветви Dв на эффективность процесса резания проведена серия из десяти компьютерных экспериментов, в которой изменяли Dв от 20 до 200 мм с шагом 20 мм.

Во всех компьютерных экспериментах скорость подачи диска составляла $30 \mathrm{~mm} / \mathrm{c}$.

При малых диаметрах ветвей (в частности при Dв $=60$ мм) пильный диск не испытывает существенного сопротивления со стороны ветви. Скорость вращения диска практически не изменяется (рис. 3, а), возникают лишь незначительные колебания зависимости $\omega(t)$, связанные с наличием отрицательной обратной связи в моделируемой системе. Давление на гидромоторе существенно не повышается (рис. 4, а), так как пильный диск практически не встречает сопротивления.
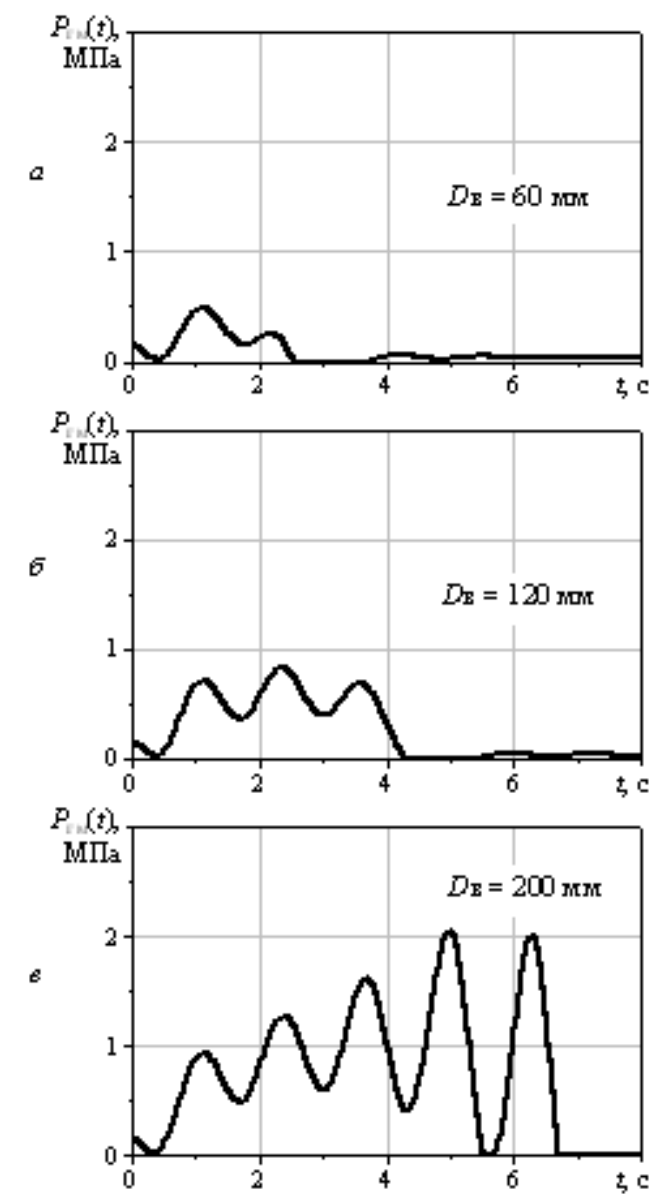

Рис. 4. Зависимости давления рабочей жидкости в гидромоторе Ргм, от времени при различных диаметрах ветви

В случае больших диаметров ветвей (для примера Dв $=200$ мм) зависимость давления рабочей жидкости в гидромоторе Ргм, уже не является плавной: на ней наблюдаются существенные осцилляции (рис. 4, б). Осцилляции на этой зависимости связаны с тем, что, встречая существенное сопротивление пильный диск замедляет вращение, что приводит к росту давления на гидромоторе и ускорению раскрутки диска; в свою очередь увеличение угловой скорости диска приводит к снижению давления на гидромоторе и повышению силы сопротивления, что приводит к уменьшению скоро- 


\section{Технологии. Машины и оборудование}

сти вращения диска. Возникает автоколебательный процесс, из-за которого наблюдаются значительные осцилляции зависимостей $\omega(\mathrm{t})$ (рис. 3, б).

Максимальная сила на зубе диска практически не зависит от диаметра ветви вплоть до $D_{\mathrm{B}}=160$ мм (рис. $5, a$ ).
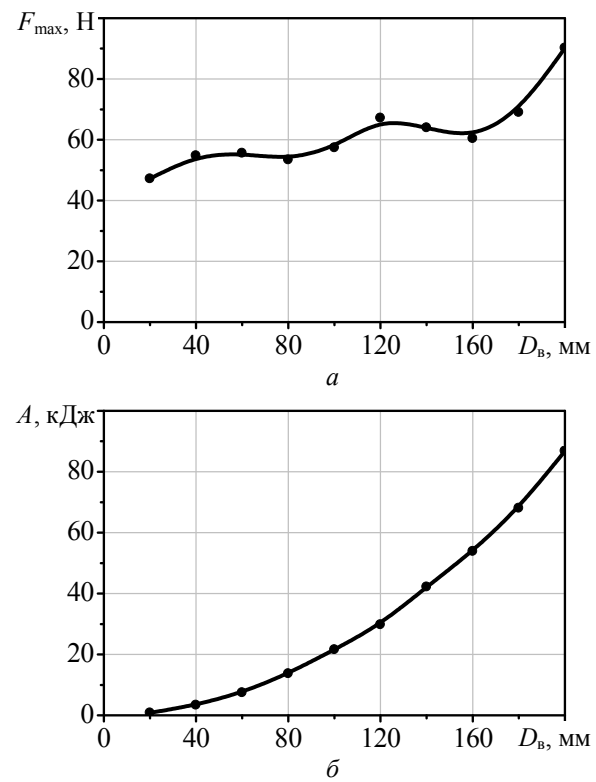

Рис. 5. Влияние диаметра ветви $D_{\text {в }}$ на максимальную силу на зубе $F_{\max }(a)$ и работу срезания ветви $A(\sigma)$
При диаметрах более 160 мм начинается резкий рост $F_{\max }$ из-за резкого роста амплитуды автоколебаний. Поэтому, можно сделать вывод, что в широком диапазоне диаметров ветвей дисковая пила с гидроприводом обеспечивает максимальную силу на зубе около $60 \mathrm{H}$ (при скорости подачи диска $30 \mathrm{~mm} / \mathrm{c}$ и частоте вращения диска 15 об/с).

С увеличением диаметра ветви $D_{\text {в }}$ работа по срезанию ветви растет приблизительно по квадратичному закону, так как площадь сечения ветви квадратично зависит от $D_{\text {в }}$ (рисунок 5 б). Существенных отклонений от квадратичной за-

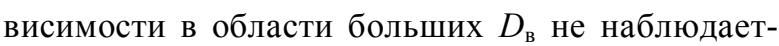
ся.Таким образом, дисковая пила с гидроприводом обеспечивает эффективное резание ветвей в широком диапазоне их диаметров (от 0 до 160 мм). В случае необходимости можно выполнять резание ветвей и больших диаметров от 160 до 300 мм, однако при этом необходимо существенно снижать скорость подачи диска.

Экспериментальные исследования подтверждают теоретические зависимости.

\section{Библиографический список}

1. Абдразаков, Ф. К. Интенсификация технологий и совершенствование технических средств в мелиоративном производстве [Текст]: монография / Ф. К. Абдразаков. - Саратов, 2002. - 350 с.

2. Афоничев, Д.Н. Манипулятор для сельскохозяйственных тракторов [Текст] / Д.Н. Афоничев, В.П. Гребнев, О.И. Поливаев // Наука вчера, сегодня, завтра материалы научно-практической конференции. - 2016. - С 76-80.

3. Бартенев, И. М. Машины и механизмы для рубок ухода: современный технический уровень [Текст] / И. М. Бартенев, Г. Л. Котляр // Лесное хозяйство. - 1992. - № 2-3. - С. 48-50.

4. Бартенев, И. М. Экологизация технологий и машин лесного комплекса [Текст] / И. М. Бартенев, С. А. Родин; ВНИИЛМ. - Пушкино, 2000. - 87 с.

5. Жданов, Ю.М. Технологии и средства механизации агролесомелиоративных работ [Текст] / Ю.М. Жданов, И. М. Бартенев // Волгоград, ВНИАЛМИ, 2011. - С. 148-168.

6. Попиков, П. И. Механизация обрезки крон деревьев в лесных насаждениях [Текст] / П. И. Попиков, Л. Д. Бухтояров, В. П. Попиков, Д. А. Азаров // Лесотехнический журнал. - 2012. - № 1 (5). - С. 71-76.

7. Пат. 2374824 РФ, МПК А01G3/00. Рабочий орган машины для подрезки крон деревьев [Текст] / В. П. Попиков, М. В. Драпалюк, Л. Д. Бухтояров ; заявитель и патентообладатель ВГЛТА. - № 2008106730/12 ; заявл. 21.02 .2008 ; опубл. 10.12.2009

8. Solodenkov, S.V. Russ. Increasing the stability of constant-speed hydromechanical systems [Text] / S.V. Solodenkov, K.I. Lyutin, E.E. Chugunova// Russian Engineering Research. - 2013. - Vol. 33, no. 9. - pp. 505-508.

9. Drapalyuk, M. V. Modeling the digging process of tree root system by the mechanism with hydropulse drive [Text] / M.V. Drapalyuk, P. I. Popikov, R.V. Yudin, A. A. Fomin, R.V. Chernukhin // IOP Conference Series: Materials Science and Engineering. - 2016. - Vol. 142. -8 p.

10. Fomin, A. A. Mechanical Treatment of Raw Waste Lumber an Effective Way to Preserve the Ecology and Resources [Text] / A. A. Fomin, V. G. Gusev, R.V. Yudin, N. F. Timerbaev, O. Y. Retyunskiy // IOP Conference Series: Materials Science 
and Engineering. - 2016. - Vol. 142(1). -8 p.

11. Zelikov, V. A. Substantiation based on simulation modeling of hitch for tillage tools parameters [Text] / V. A. Zelikov, V. I. Posmetiev, M. A. Latysheva // World Applied Sciences Journal. - 2014. Vol. -30, no. 4. - pp. 486-492.

\section{References}

1. Abdrazakov F.K. Intensifikatsiya teknologiy i sovershenstvovanie tekhnicheskikh sredstv v meliorativnom proizvodstve. [Intensification of technology and the improvement of technical equipment in the production of ameliorative]. Saratov, 2002, 350 p. (In Russian).

2. Afonichev D.N., Grebnev V.P., Polivaev O.I. Manipulyator dlya sel skokhozyaystvennykh traktotov [Manipulator for agricultural tractors]. Nauka vchera, segodnya, zavtra materialy nauchno-prakticheckoy konferentsii [Science yesterday, today, tomorrow. Proceddings of the Conference] 2016, pp. 76-80. (In Russian).

3. Bartenev I.M., Kotlyar G.L. Mashiny i mekhanizmy dlya rubok ukhoda: sovremennyy tekhnicheskiy uroven '[Machines and mechanisms for thinning: state of the art]. Lesnoe khozyaystvo [Forestry], 1992, no. 2-3, pp. 48-50. (In Russian).

4. Bartenev LA., Rodin M.S. Ekologizatsiya tekhnologiy i mashin lesnogo kompleksa [Greening of technologies and machines forest complex]. Pushkino, 2000, 87 p. (In Russian).

5. Zhdanov Yu.M., Bartenev I.M. Tekhnologii i sredstva mekhanizalsii agrolesomeliora-livnykh robot [Technologies and means of mechanization agroforestry works]. Volgograd, 2011, pp. 148-168. (In Russian).

6. Popikov P.I., Bukhtoyarov L.D,. Popikov V.P, Azarov D.A. Mekhanizatsiya obrezki kron derev'ev v lesnykh nasazhdeniyakh [Mechanization of pruning trees in forest plantations] Lesotekhnicheskii zhurnal, 2012, no. 1 (5), pp. 71-76. (In Russian).

7. Popikov P.I., Bukhtoyarov L.D., Venevitin A.A., V.P. Popikov Rabochiy organ mashiny dlya obrezki kron derev'ev [Operating the machine body for pruning trees]. Patent RF, no. 2014152750/13, 2016. (In Russian).

8. Solodenkov S.V., Lyutin K.I., Chugunova E.E. Increasing the stability of constant-speed hydromechanical. Russian Engineering Research, 2013, Vol. 33, no. 9, pp. 505-508.

9. Drapalyuk M. V., Popikov P.I., Yudin R.V., Fomin A.A., Chernukhin R.V. Modeling the digging process of tree root system by the mechanism with hydropulse drive. IOP Conference Series: Materials Science and Engineering, 2016, Vol. 142, 8 p.

10. Fomin A. A., Gusev V.G., Yudin R.V., Timerbaev N.F., Retyunskiy O.Y. Mechanical Treatment of Raw Waste Lumber an Effective Way to Preserve the Ecology and Resources. IOP Conference Series: Materials Science and Engineering, 2016, Vol. 142(1), 8 p.

11. Zelikov V. A. Posmetiev V.I., Latysheva M.A. Substantiation based on simulation modeling of hitch for tillage tools parameters. World Applied Sciences Journal, 2014. Vol. 30, no. 4, pp. 486-492.

\section{Сведения об авторах}

Бухтояров Леонид Дмитриевич - заведующий кафедрой лесной промышленности, метрологии, стандартизации и сертификации ФГБОУ ВО «Воронежский государственный лесотехнический университет имени Г.Ф. Морозова», кандидат технических наук, доцент, г. Воронеж, Российской Федерации; e-mail: vglta-mlx@yandex.ru

Попиков Виктор Петрович - доцент кафедры ландшафтной архитектуры и почвоведения ФГБОУ ВО «Воронежский государственный лесотехнический университет имени Г.Ф. Морозова», кандидат технических наук, г. Воронеж, Российской Федерации; e-mail: vglta-mlx@yandex.ru

\section{Information about authors}

Bukhtoyarov Leonid Dmitrievich - Head of Department of Forest Industry, Metrology, Standardization and Certification department, Federal State Budget Education Institution of Higher Education «Voronezh State University of Forestry and Technologies named after G.F. Morozov»», Associate Professor, Ph.D. in Engineering, Voronezh, Russian Federation; e-mail: vgltamlx@yandex.ru

Popikov Viktor Petrovich - Associate Professor of Landscape Architecture and Soil Science department, Federal State Budget Education Institution of Higher Education «Voronezh State University of Forestry and Technologies named after G.F. Morozov», Ph.D. in Engineering, Voronezh, Russian Federation; e-mail: vglta-mlx@yandex.ru 